Stosic, D. (2012), « Le pouvoir cadratif des compléments introduits par à travers: des cadres de discours pas comme les autres? ». Travaux de linguistique $\mathrm{n}^{\circ} 64$, p. 55-78.

\title{
Le pouvoir cadratif des compléments introduits par à travers : des cadres de discours pas comme les autres?
}

Dejan STOSIC (Université d'Artois, Grammatica EA 4521)

\section{Introduction $^{1}$}

Un des objectifs du projet $\mathrm{SFA}^{2}$ était de contraster des adverbiaux introduits par des prépositions spatiales statiques (ex. à, dans, en) et ceux introduits par des prépositions dynamiques (ex. depuis, par, vers) selon leur capacité : i) à apparaître en position initiale de la phrase et ii) à introduire des cadres de discours au sens de Charolles (1997). De nombreux travaux ont en effet permis de montrer que les syntagmes prépositionnels (SP) formés à partir des prépositions statiques (ex. en France, au Japon, dans le salon) remontent facilement en tête de phrase et étendent souvent leur portée sémantique au-delà de leur phrase d'accueil en regroupant les propositions indexées dans des unités discursives appelées cadres de discours (voir entre autres Charolles 1997, Charolles \& Péry-Woodley (éds) 2005, Charolles, Le Draoulec, Pery-Woodley \& Sarda 2005, Prévost 2003, Sarda 2005). Quant aux compléments spatiaux dynamiques, il est généralement admis que leur détachement en position initiale est plus rare et qu'ils n'ont quasiment pas la capacité à fonctionner au niveau discursif comme cadratifs. Force est cependant de constater que des études qui traitent du pouvoir cadratif des adverbiaux spatiaux dynamiques sont très rares (voir Papahagi 2005, Sarda \& Stosic 2007, Lagae à paraître).

L'objectif de notre article sera de faire une étude sur corpus du fonctionnement intraphrastique et discursif des syntagmes prépositionnels introduits par à travers, qui est un marquer spatial dynamique. Pour les besoins de cette étude, nous nous focaliserons principalement sur des énoncés où un SP introduit par à travers figure en tête de phrase, cette position étant cruciale pour le déclenchement d'un cadre de discours. Dans la première section, nous donnons une description sommaire du sémantisme de la préposition à travers, en mettant un accent particulier sur ses emplois spatiaux. La deuxième section explore les facteurs syntaxiques et sémantiques qui favorisent la remontée en tête de phrase des SPrép en à travers. Enfin, dans la troisième section, nous montrons que les compléments introduits par à travers peuvent, sous certaines conditions, introduire des cadres de discours en dépit du caractère dynamique de leur sémantisme. Ces cadres se distinguent cependant en de

\footnotetext{
${ }^{1}$ Nous remercions les relecteurs anonymes pour leurs remarques et commentaires qui nous ont permis d'améliorer considérablement notre article.

${ }^{2}$ Le projet Spatial Framing Adverbials (2006-2009), dirigé par M. Charolles et L. Sarda (LATTICE, UMR 8094) a été financé par le programme «blanc» 2006 de l’Agence Nationale pour la Recherche (ANR).
} 
Stosic, D. (2012), « Le pouvoir cadratif des compléments introduits par à travers: des cadres de discours pas comme les autres? ». Travaux de linguistique $\mathrm{n}^{\circ} 64$, p. 55-78.

nombreux points de ceux initiés par les adverbiaux spatiaux statiques, le rôle des adverbiaux en $\grave{a}$ travers étant principalement de donner accès à une scène qui, par ce fait même, pourra faire l'objet d'un développement dans la partie indexée par l'élément introducteur.

Précisons enfin que la présente étude s'appuie sur un ensemble de 600 énoncés attestés où un complément spatial introduit par à travers figure en tête de phrase. Puisque la base constituée dans le cadre du projet $\mathrm{SFA}^{3}$ n'a pas pu nous fournir un corpus suffisamment large, nous avons extrait des données supplémentaires de Frantext en nous limitant à la période 1900-2007.

\section{La préposition à travers en français}

Cette section a pour but de donner un bref aperçu du fonctionnement sémantique de la préposition à travers en français, aperçu qui sera basé sur des résultats des travaux qui y ont été consacrés les trois dernières décennies. Ce survol nous semble indispensable dans la mesure où nous montrerons dans la dernière partie de l'article que le comportement discursif des compléments en à travers est fortement tributaire de son sémantisme.

\subsection{La polysémie de à travers}

Il est possible de distinguer dans le cas de à travers trois grands types d'emplois : a) les emplois spatiaux, comme en [1]-[2], b) les emplois temporels, en [3]-[4] et c) les emplois instrumentaux, illustrés dans les exemples [5]-[6] :

[1] Malgré leur fatigue, les hommes et les femmes ont cheminé pendant des semaines à travers les montagnes rouges, le long des torrents sans eau. (Le Clézio, Le bonheur)

[2] L'œil affûté, elle m'aperçut tout de suite, s'approcha de moi et me prit la main à travers les barreaux. (Jardin, Bille en tête)

[3] Son (=Lénin) nom vivra à travers les siècles, son œuvre aussi. (Mordillat, Vive la sociale!)

[4] Le droit a beaucoup évolué à travers les âges. (Le Monde)

[5] J'oubliais dans mon aberration que la vie n'est pas faite pour la jouissance facile, mais pour un labeur et un perfectionnement qui ne s'atteignent qu'à travers des efforts et des contraintes. (Havet, Journal 1919-1924)

[6] À travers son œuvre, l'écrivain livre une vision de l'homme et du cosmos. (Le Monde)

Ces exemples montrent bien que le sémantisme des éléments du contexte (linguistique) joue un rôle déterminant dans l'orientation de l'interprétation vers l'une des acceptions mentionnées ci-dessus. Ainsi, avec des SN renvoyant à des entités spatiales - lieux, objets, substances (ex. la forêt, la fenêtre, le tunnel, le brouillard, etc.), à travers aura une interprétation spatiale, alors qu'avec des $\mathrm{SN}$ comme le temps, les siècles, l'histoire, etc., à

\footnotetext{
${ }^{3}$ Pour plus de détails sur le corpus constitué et exploité dans le cadre du projet SFA, voir la présentation de ce numéro.
} 
Stosic, D. (2012), « Le pouvoir cadratif des compléments introduits par à travers: des cadres de discours pas comme les autres ? ». Travaux de linguistique $\mathrm{n}^{\circ} 64$, p. 55-78.

travers se prêtera facilement à une interprétation temporelle. Combiné aux SN renvoyant à des abstractions (ex. l'obscurité de son propos, la continuité, sa grâce, leur puissance, etc.), à des idéalités (ex. ce récit, son cuvre, cette sonate, ce tableau, etc. ; cf. Husserl 1970a, b, Flaux et Stosic 2011, Stosic et Flaux 2012), ou aux individus (ex. lui, cette femme, les otages, un interprète), à travers construit, en règle générale, des sens instrumentaux. Dans ce dernier type d'emploi, à travers est paraphrasable, selon le contexte, par par le truchement de, à l'aide de, grâce à ou par, et introduit un complément qui exprime un moyen, une technique ou un fait qui rend possible la réalisation du procès décrit par le verbe. Le sens du verbe peut, lui aussi, imposer telle ou telle interprétation; même si dans les exemples [7] et [8], à travers est accompagné d'un même SN (les ruelles), dans le premier cas on a un emploi spatial, dans le second un emploi instrumental :

[7] Par un clair après-midi de mai, elle marchait à travers les ruelles de la Cité.

[8] Nous étions ressortis, et mon compagnon paraissait savoir quel était mon hôtel, car il nous menait dans sa direction, pour autant que j'en pouvais juger à travers les ruelles que nous enfilions. (Tournier, Les Météores)

La préposition à travers connaît également de nombreuses extensions figurées, dont voici quelques exemples :

[9] L'exposition invite à un voyage à travers l'amour. (Le Monde)

[10] Il y a un fil unique qui court à travers l'existence. (D'Ormesson, La Douane de mer)

[11] À travers les orages qui vous environnent, voyez les beaux jours que vous préparez aux races futures ; la liberté du monde entier sera votre récompense. (Simon, Les Géorgiques)

En [9] par exemple, la relation exprimée par à travers est tout à fait analogue à celle en [7], à cette différence près que le domaine auquel elle s'applique n'est pas celui de l'espace, mais celui, plus abstrait, de l'amour.

Même si nous ne pourrons pas entrer ici dans les détails du problème, notons que la possibilité pour à travers, comme en [12]-[13], d'admettre la coordination de SN renvoyant à deux domaines notionnels distincts appuie fortement l'hypothèse de l'existence d'un lien entre les différents sens de à travers :

[12] J'essayais surtout de comprendre - et c'était une tâche infinie - ce qui différenciait, à travers l'espace et le temps, les régions et les âges. (D'Ormesson, Le Vent du soir)

[13] Ce n'est pas de moi que je parle dans ces pages, c'est d'un être assez curieux, curieux, divers et multiple et qui avait pourtant son unité, à la fois exceptionnel et banal, qui entretenait avec son temps des rapports exemplaires et ambigus, et dont j'essaie de retracer, à travers les années et les individus, les idées collectives et l'évolution - je veux dire : ma vieille et chère famille. (D’Ormesson, Au plaisir de Dieu) 
Stosic, D. (2012), « Le pouvoir cadratif des compléments introduits par à travers: des cadres de discours pas comme les autres? ». Travaux de linguistique $\mathrm{n}^{\circ} 64$, p. 55-78.

Les données attestées semblent en effet suggérer que le caractère médian des relations exprimées par à travers et sa dynamicité impliquant le parcours de l'entité dénotée par le SN complément, constituent les principaux traits sémantiques à l'origine de sa polysémie.

Puisque notre objectif est de vérifier le pouvoir cadratif des adverbiaux spatiaux formés à l'aide de la préposition à travers, nous nous focaliserons dans le paragraphe suivant sur son sens spatial.

\subsection{Le sens spatial de la préposition à travers}

Les usages spatiaux de la préposition à travers sont loin d'être homogènes. Plusieurs études qui traitent du sens spatial de à travers, ont montré que, dans ses emplois spatiaux, $\grave{a}$ travers décrit le plus souvent le déplacement [14]-[15], mais il peut aussi exprimer un type particulier de localisation statique, qualifiée de « balayage » par Stosic (2002) [16]-[17], et la perception [18]-[20] (voir, entre autres, Kwon-Pak 1997, Aurnague 2000, Stosic 2002, Dominicy \& Martin 2005).

[14] Les Filles du Feu passaient à travers des cerceaux enflammés, changeaient de trapèze tout en jonglant avec des torches... (Perec, La Vie mode d'emploi)

[15] Des enfants jouaient avant le dîner, et se poursuivaient à travers l'herbe trempée à grand bruit de sabots. (Gracq, La Presqu'île)

[16] Il y avait, à la cour de France, des femmes que leur beauté avait rendues célèbres à travers le monde entier (D'Ormesson, La Douane de mer).

[17] Le métier, l'argent, l'amour, l'aventure nous avaient éparpillés à travers l'univers. (D'Ormesson, Au plaisir de Dieu)

[18] Il se releva brusquement, referma le lit, revint vers la fenêtre et regarda de nouveau à travers les volets. (Moinot, Le Guetteur d'ombre)

[19] On entendait à travers le mur une voix douce et posée.

[20] Il faisait beau, une lumière très blanche, mais je sentais l'air froid à travers le carreau. (Djian, $37^{\circ} 2$ le matin)

Pour notre propos, il est important de souligner que la préposition à travers possède un contenu sémantique dynamique, et appartient, par sa polarité, à la sous-classe des prépositions de polarité médiane (cf. Laur 1993, Borillo 1998, Stosic 2002). Cela signifie qu'elle exprime des relations spatiales dynamiques qui se vérifient pendant la phase médiane du déplacement, i.e. pendant la phase du parcours. Les prépositions de polarité médiane (ex. par, à travers, autour de, le long de) s'opposent à celles de polarité initiale (ex. de, depuis) et finale (ex. jusque, vers), qui insistent respectivement sur le point de départ et le but du déplacement.

En ce qui concerne la définition du sémantisme de la préposition à travers, les travaux traitant de ses emplois spatiaux proposent des concepts à la fois nombreux et variés. Nous nous contenterons ici d'en présenter brièvement quelques-uns. 
Stosic, D. (2012), « Le pouvoir cadratif des compléments introduits par à travers: des cadres de discours pas comme les autres? ». Travaux de linguistique $\mathrm{n}^{\circ} 64$, p. 55-78.

La notion d' « obstacle » réapparaît le plus souvent dans les descriptions du sens spatial de à travers et elle va de pair, en règle générale, avec la notion d'« opposition/résistance au mouvement » (cf. Spang-Hanssen 1963, Kwan-Pak 1997, TLFi). Les auteurs retenant ces notions postulent que la préposition à travers décrit des situations où le site présente un obstacle au déplacement, c'est-à-dire qu'il résiste au passage de la cible. Nous avons montré ailleurs (cf. Stosic 2002, 2007b) que la notion d'obstacle pose de nombreux problèmes lorsqu'on la met à l'épreuve des données. En effet, même si à travers décrit incontestablement des situations où l'entité parcourue - désormais le site - entrave le déplacement de l'entité en mouvement - désormais la cible ${ }^{4}$ - comme en [21], ce n'est pas le cas dans tous ses emplois. En [22], par exemple, le site ne présente pas d'éléments susceptibles de s'opposer au passage de la cible, tout comme en [23], où le site est une entité fonctionnellement destinée plutôt à faciliter le déplacement :

[21] Certains seraient capables de faire plus de $200 \mathrm{~km}$ à pied à travers la forêt.

[22] Les armes volèrent à travers la clairière vide jusqu'au bois, et là, elles trouvèrent leur cible. (Internet)

[23] L'eau s'écoule à travers le tuyau.

Ajoutons que la notion d'obstacle souffre également de son utilisation intuitive et de son caractère peu discriminant dans la mesure où, telle quelle, elle pourrait tout aussi bien s'appliquer à la description des usages spatiaux de contre ou de par-dessus. Il est en effet bien connu (cf. entre autres, Dendale 2001, Vandeloise 2003, 2005, Borillo 2007), que certains déplacements décrits par contre peuvent se résoudre par un contact violent et donc être arrêtés par le site, comme dans l'exemple suivant :

[24] Un petit avion s'est écrasé mercredi contre un immeuble résidentiel de 50 étages.

Or, dans les configurations spatiales décrites par à travers, le site n'arrête pratiquement jamais la cible ; même si certains mouvements de la cible sont contraints, les mouvements frontaux restent, en général, possibles et la cible arrive toujours à avancer et/ou à passer par l'intérieur du site. Par conséquent, même si le trait d'opposition au mouvement joue un rôle important dans la sémantique de la préposition à travers, il ne peut pas à lui seul rendre compte de son fonctionnement ${ }^{5}$.

Plutôt sceptique vis-à-vis de la notion d'« obstacle », Flageul (1997 : 221) attribue deux valeurs sémantiques à à travers spatial : celle de « trajectoire dans l'intérieur » qui insiste sur la construction d'une trajectoire au sein du lieu parcouru (ex. se promener à travers la ville),

\footnotetext{
${ }^{4}$ Nous empruntons les termes bien connus « cible » et « site » à Vandeloise (1986).

${ }^{5}$ Pour une remise en cause plus argumentée de la notion d'« obstacle», voir Stosic (2002: 94-102) ou Stosic (2007b).
} 
Stosic, D. (2012), « Le pouvoir cadratif des compléments introduits par à travers: des cadres de discours pas comme les autres? ». Travaux de linguistique nº64, p. 55-78.

et celle de "franchissement», qui intervient dans des cas où l'extension du site est insuffisante pour qu'on puisse parler de la construction d'une trajectoire (ex. sauter à travers le cerceau). Le repérage par rapport à l'intérieur impliquant une réelle traversée du site semble donc fondamental pour la sémantique de à travers d'après Flageul (1997).

Pour Plungian (2002), la «dominante sémantique » de la préposition à travers est « l'accès à ou par la structure intérieure », alors que Weinrich (1989) fait appel à la notion de « progression ». D'après ce dernier : «Au lieu d'embrasser l'ensemble d'un processus d'un même regard (...), la préposition à travers désigne un processus en faisant abstraction du début et de la fin. Elle n'envisage que le processus linéaire tout court : nous en décrivons donc le sens par le trait sémantique de 'PROGRESSION' ( (Weinrich 1989 : 409).

En contrastant les emplois perceptifs et spatiaux de à travers et de au travers de, Dominicy \& Martin (2005) proposent la notion d'« orthogonalité » pour décrire à travers. Selon ces auteurs, la conceptualisation des situations décrites par à travers repose essentiellement sur la décomposition du site en une série de «tranches successives » qui entrent nécessairement dans un « rapport d'orthogonalité » soit vis-à-vis d'un segment de la trajectoire soit vis-à-vis de l'ensemble du chemin parcouru par la cible (ex. rouler à travers les routes sinueuses de compagne). Notons que, d'après les auteurs, la notion « orthogonalité » met au premier plan l'orientation frontale.

Enfin, après avoir examiné en détail la pertinence de ces notions en les mettant à l'épreuve d'un vaste corpus d'énoncés attestés, nous avons proposé, en collaboration avec M. Aurnague, la notion de « guidage » pour définir le sens spatial de à travers (cf. Stosic 2002, 2007b, Aurnague 2000). La notion de «guidage » est un concept sémantique complexe qui regroupe, sous forme d'une ressemblance de famille, sept traits sémantiques dont les combinaisons permettent à la fois de rendre compte de l'ensemble des usages spatiaux acceptables de à travers, d'exclure ses usages inacceptables et de l'opposer à plusieurs marqueurs plus ou moins concurrents (ex. par, par-dessus, parmi, contre). En proposant la concept de " guidage », il ne s'agissait en aucun cas pour nous de rejeter les notions élaborées par d'autres auteurs. En effet, même si aucune d'entre elles n'est entièrement satisfaisante pour la description du sens spatial de à travers, elles mettent en évidence, chacune à sa façon, différentes propriétés sémantiques de cette préposition. C'est pourquoi la plupart de ces notions se retrouvent, moyennant une définition plus rigoureuse, dans le concept de « guidage ». Plus précisément, nous avons mis au jour dans Stosic (2002) puis repris et affiné dans Stosic (2009) les traits sémantiques suivants : « dynamicité », « intériorité », « unicité du site », « opposition au mouvement», « orientation latérale », « minimum de parcours », 
Stosic, D. (2012), « Le pouvoir cadratif des compléments introduits par à travers: des cadres de discours pas comme les autres ? ». Travaux de linguistique $\mathrm{n}^{\circ} 64$, p. 55-78.

« focalisation sur le parcours du site ». D’après cette analyse, qui se situe dans le cadre de la sémantique du prototype, aucun de ces traits n'est ni nécessaire ni suffisant. Le noyau des usages prototypiques de à travers vérifie l'ensemble ou la quasi-totalité des traits de la ressemblance de famille tandis que les usages «marginaux» peuvent être expliqués par diverses combinaisons des traits en question. Ainsi, dans des situations prototypiques décrites à l'aide de à travers le site réalise, par ses frontières latérales et/ou par sa structure interne, une certaine forme de contrôle de la position de la cible essentiellement sur l'axe latéral, les mouvements frontaux restant, en principe, possibles. La trajectoire du déplacement se situe, en entier ou en partie, à l'intérieur du site et doit avoir une extension significative par rapport à la totalité de sa surface : plus le parcours est long et étendu, plus grande est la probabilité d'utiliser à travers pour décrire le déplacement en question. Le franchissement du site est possible mais n'est aucunement indispensable, le déplacement pouvant s'effectuer entièrement au sein de l'entité parcourue.

Il suit de ce qui précède que à travers apparaît, dans l'expression de l'espace, comme une préposition intrinsèquement dynamique qui implique le parcours effectif ${ }^{6}$ de $1^{\prime}$ 'entité localisatrice. Dans la section suivante, nous examinons dans quelle mesure les SP introduits par un tel marqueur peuvent être détachés en tête de phrase, cette position étant cruciale pour qu'un adverbial puisse ouvrir un cadre de discours.

\section{Le détachement en tête de phrase des SP formés à l'aide de à travers}

Comme nous l'avons précisé dans l'introduction, l'analyse du fonctionnement intraphrastique et discursif des SP introduits par à travers en tête de phrase s'appuie sur un corpus de 600 énoncés attestés présentant cette structure. Le premier point à noter est que les compléments en à travers apparaissent très rarement en position initiale. En effet, sur un ensemble de 17000 occurrences de à travers relevées dans Frantext pour la période retenue, on n'en trouve qu'environ 900 en tête de phrase, ce qui représente une portion très faible $(5 \%)$.

\footnotetext{
${ }^{6}$ Sur ce point, à travers s'oppose à par qui n'impose pas la contrainte de parcours effectif. Il est en effet tout à fait courant de dire lors des déplacements en avion: Pour aller à New York, j'étais obligé de passer par Amsterdam. Sans que la ville d'Amsterdam soit effectivement parcourue, elle fournit un jalon de parcours (pour plus de détails sur la sémantique de par, voir Aurnague 2000, Stosic 2002, 2007a, Aurnague et Stosic 2002). En pareil contexte, l'emploi de à travers n'est pas possible : *Pour aller à New York, j'étais obligé de passer à travers Amsterdam.
} 
Stosic, D. (2012), « Le pouvoir cadratif des compléments introduits par à travers: des cadres de discours pas comme les autres ? ». Travaux de linguistique $\mathrm{n}^{\circ} 64$, p. 55-78.

Ce corpus nous a permis tout d'abord d'examiner quels types d'emplois remontent le plus souvent en tête de phrase. Lors de l'analyse, nous avons pu identifier les usages spatiaux [25], perceptuels ${ }^{7}$ [26], instrumentaux [27], temporels [28] et métaphoriques [29]:

[25] À travers le Turkestan, l'Afghanistan et le Penjab, les pèlerins visitèrent les principaux lieux saints du bouddhisme. (Daumas, éd., Histoire de la science)

[26] À travers les rideaux de macramé, il aperçut la mercière qui lisait. (Sabatier, David et Olivier)

[27] À travers le regard de ce curé de campagne, on suit, au jour le jour, la vie des villageois, les moments d'espoir, les combats et les habitudes de l'occupant. Recensements, impôts en tous genres, départ des prisonniers, tout est consigné. (Le Monde)

[28] À travers les quarante années que jalonnent ces étapes, un même sentiment reste constant : le désir de la fuite, du refuge, la nostalgie d'un paradis perdu qui prend des formes diverses. (Béguin, L'Âme romantique et le rêve)

[29] À travers les récits de Victor Serge passaient, par centaines, par milliers, par centaines de milliers, des fantômes affamés et décharnés. (D’Ormesson, Le Bonheur à San Miniato)

Il apparaît, selon nos données, que ce sont les compléments locatifs accompagnant des verbes de perception visuelle et auditive qui apparaissent le plus souvent en position initiale, dans $38 \%$ des cas. Viennent ensuite les emplois instrumentaux de à travers avec une fréquence de $33 \%$ et seulement à la troisième position les emplois purement locatifs, qui ne représentent que $20 \%$ des occurrences. Pour mieux saisir les particularités de fonctionnement des SP en $\grave{a}$ travers en position initiale, nous avons vérifié la répartition de ce type de syntagmes en position postverbale sur un échantillon de 350 exemples de Frantext choisis de manière aléatoire dans un ensemble de 1300 résultats. Les tableaux qui suivent mettent clairement en évidence les différences de fonctionnement dues à la position syntaxique.

Position postverbale

\begin{tabular}{|c|c|c|}
\hline Type d'emploi & \multicolumn{2}{|c|}{ Fréquence } \\
\hline Spatial & 182 & $52 \%$ \\
\hline Instrumental & 68 & $19 \%$ \\
\hline Perceptuel & 53 & $15 \%$ \\
\hline Autres & 47 & $14 \%$ \\
\hline Total & 350 & $100 \%$ \\
\hline $\begin{array}{r}\mathbf{T} \\
\text { Répartition de } \\
\text { po }\end{array}$ & $\begin{array}{l}1 \\
\text { ois } \epsilon \\
\text { ale }\end{array}$ & ion \\
\hline
\end{tabular}

\section{Position initiale}

\begin{tabular}{|c|c|c|}
\hline Type d'emploi & \multicolumn{2}{|c|}{ Fréquence } \\
\hline Perceptuel & 228 & $38 \%$ \\
\hline Instrumental & 197 & $33 \%$ \\
\hline Spatial & 119 & $20 \%$ \\
\hline Autres & 56 & $9 \%$ \\
\hline Total & 600 & $100 \%$ \\
\hline $\begin{array}{r}\text { Ta } \\
\text { Répartition des } \\
p\end{array}$ & $\begin{array}{l}\text { au } 2 \\
\text { nploi } \\
\text { ase }\end{array}$ & de \\
\hline
\end{tabular}

On constate donc qu'en position postverbale les emplois spatiaux représentent la moitié des occurrences des SP en à travers et que les emplois perceptuels et instrumentaux sont beaucoup moins nombreux. Du point de vue sémantique, il y aurait donc une inversion de

\footnotetext{
${ }^{7}$ Pour les besoins de l'analyse, nous séparons ici les emplois perceptuels et spatiaux, mais il va de soi que les premiers font partie des seconds.
} 
Stosic, D. (2012), « Le pouvoir cadratif des compléments introduits par à travers: des cadres de discours pas comme les autres? ». Travaux de linguistique $\mathrm{n}^{\circ} 64$, p. 55-78.

tendances due au détachement à gauche du complément introduit par à travers, comme on le voit bien sur le graphique ci-dessous :

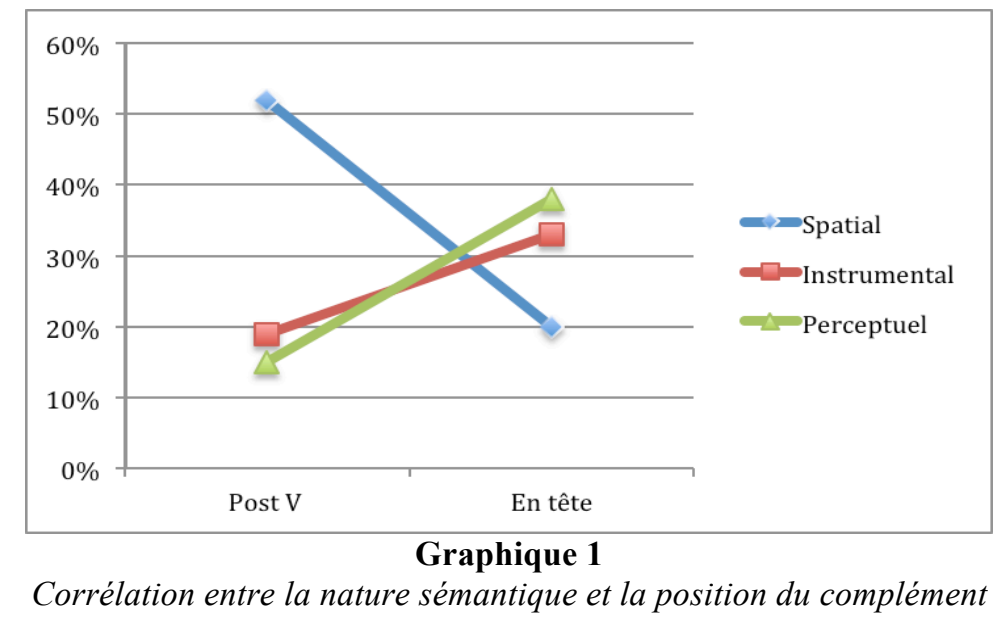

Il suit de ce qui précède que les adverbiaux spatiaux introduits par à travers, lorsqu'ils expriment la localisation au sens propre du terme et non la perception, présentent une réticence au détachement en position initiale. Sans qu'elle soit exclue, l'étendue de leur portée sémantique sur l'ensemble de la phrase se fait donc plus difficilement que dans le cas des compléments perceptuels et instrumentaux.

Au-delà d'une apparente affinité entre certains types sémantiques de compléments en $\grave{a}$ travers et la position initiale, on relève, dans le corpus, des facteurs plus strictement syntaxiques qui soit favorisent soit empêchent la remontée en tête de phrase des SP en question. La transitivité des prédicats verbaux est un de ces facteurs. En effet, nous avons pu constater que $62 \%$ des exemples avec un SP en à travers en position initiale comportent un verbe transitif direct. La grande majorité de ces verbes expriment soit la perception - dans $54 \%$ des cas (ex. voir, entendre, regarder, apercevoir), soit diverses actions dont la réalisation nécessite l'utilisation d'un moyen - dans $29 \%$ des cas (ex. découvrir, atteindre, comprendre), et très rarement le déplacement $-9 \%$ des cas (ex. traverser, atteindre, pénétrer). La transitivité directe de ces verbes libère en quelque sorte le complément prépositionnel qui, à cause de son statut nécessairement non-argumental, devient facilement détachable à l'initiale de la phrase. En revanche, le statut syntactico-sémantique des SP formés à l'aide de à travers combinés avec les verbes intransitifs et/ou transitifs indirects est plus ambigu. Faute d'études plus approfondies sur la question (voir cependant Bonami 1999 sur les SP de but locatif), nous nous contenterons de préciser que, dans ce type de contextes, les compléments en $\grave{a}$ 
Stosic, D. (2012), « Le pouvoir cadratif des compléments introduits par à travers: des cadres de discours pas comme les autres ? ». Travaux de linguistique $\mathrm{n}^{\circ} 64$, p. 55-78.

travers fonctionnent sinon comme de véritables arguments (syntaxiques et sémantiques) ${ }^{8}$, du moins comme des arguments sémantiques (ex. passer/ couper/marcher à travers la forêt, etc.). C'est certainement ce lien sémantique assez fort qui pourrait expliquer le faible taux de détachement des compléments en à travers (16\%) lorsqu'ils sont combinés avec les verbes intransitifs et/ou transitifs indirects ${ }^{9}$. Les contextes avec un verbe transitif direct favoriseraient donc la remontée en tête de phrase des compléments en à travers alors que la présence d'un verbe intransitif et/ou transitif indirect serait un facteur défavorable au détachement. Cette corrélation est d'autant plus significative qu'en position postverbale, les compléments en $a ̀$ travers accompagnent les verbes transitifs directs dans $45 \%$ des cas, et les verbes intransitifs et/ou transitifs indirects dans 39\% des énoncés analysés, ce que nous résumons dans les tableaux suivants :

\section{Position postverbale}

\begin{tabular}{|c|c|c|}
\hline Constr. synt. & \multicolumn{2}{|c|}{ Fréquence } \\
\hline Trans. directe & 157 & $45 \%$ \\
\hline Intr. \& Tr. ind. & 138 & $39 \%$ \\
\hline Pronominale & 32 & $9 \%$ \\
\hline Autres & 23 & $7 \%$ \\
\hline Total & 350 & $100 \%$ \\
\hline
\end{tabular}

Tableau 3

Répartition des verbes selon leur transitivité en position postverbale

\begin{tabular}{lrr}
\multicolumn{3}{c}{ Position initiale } \\
Constr. synt. & \multicolumn{2}{c}{ Fréquence } \\
\hline Trans. directe & 371 & $62 \%$ \\
Intr. \& Tr. ind. & 99 & $16 \%$ \\
Pronominale & 66 & $11 \%$ \\
Autres & 64 & $11 \%$ \\
\hline \multicolumn{1}{r}{ Total } & 600 & $100 \%$
\end{tabular}

Tableau 4

Répartition des verbes selon leur transitivité en tête de phrase

Même si dans les deux positions, ce sont les verbes entrant dans une structure transitive directe qui introduisent le plus souvent les compléments en à travers, on constate un creusement assez net de l'écart entre la construction transitive directe d'une part et la construction intransitive et/ou transitive indirecte d'autre part.

\section{Le pouvoir cadratif des adverbiaux spatiaux introduits par à travers}

Comme nous l'avons dit au tout début, notre analyse se situe dans la perspective de l'encadrement du discours, modèle initialement développé par M. Charolles (cf. Charolles 1997). Avant d'aborder le fonctionnement discursif des adverbiaux en à travers, nous

\footnotetext{
${ }^{8}$ Nous empruntons cette opposition entre arguments syntaxiques et arguments sémantiques à Bonami (1999). Comme le prédit la tradition logique et linguistique, tout véritable argument est sélectionné syntaxiquement et sémantiquement par le prédicat. Il existe cependant des cas où un dépendant est sélectionné sémantiquement sans être obligatoire du point de vue syntaxique, ce dont vise à rendre compte l'opposition entre arguments syntaxiques (les vrais arguments) et sémantiques (sélectionnés sémantiquement mais pas syntaxiquement).

${ }_{9}$ Nous faisons exprès de ne pas distinguer les verbes entrant dans une structure intransitive et ceux qui apparaissent dans la structure transitive indirecte, nombreux étant des cas où, faute d'études plus approfondies, il est difficile de faire une opposition nette. On peut se demander, en effet, si les verbes comme passer, arriver, partir, etc. sont intransitifs ou bien transitifs indirects, les dictionnaires et les linguistes en donnent souvent des caractérisations différentes.
} 
Stosic, D. (2012), « Le pouvoir cadratif des compléments introduits par à travers: des cadres de discours pas comme les autres ? ». Travaux de linguistique $\mathrm{n}^{\circ} 64$, p. 55-78.

présentons, dans la section suivante, les grandes lignes du cadre théorique adopté. L’introduction à ce numéro comporte une présentation plus détaillée du modèle de Charolles.

\subsection{Cadre théorique : l'encadrement du discours (Charolles 1997)}

La modèle de l'encadrement du discours met en avant la possibilité qu'ont certains adverbiaux d'étendre leur portée au-delà de la phrase où ils apparaissent. Dans l'exemple [30] ci-dessous, tiré d'un livre pour enfants, les adverbiaux En automne et En hiver découpent respectivement deux segments de texte en étendant leur portée sur plusieurs propositions :

[30] [En automne, les journées raccourcissent et le temps se rafraîchit. La nature prend de belles couleurs marron, jaune et rouge. Le vent souffle souvent et emporte les feuilles mortes. Beaucoup d'oiseaux s'envolent vers des pays plus chauds. Les animaux se préparent aux grands froids et font leurs provisions de nourriture.]

[En hiver, les nuits sont plus longues que les jours. Le temps refroidit et la nature vit au ralenti. Dans le potager, rien ne pousse. Presque tous les arbres perdent leurs feuilles. Les animaux trouvent peu de choses à manger. Le lièvre se contente de brindilles ou d'écorces d'arbres. Sur les pentes enneigées, on peut faire du ski, du surf ou de la luge.] (Girard \& Fordacq, Les saisons)

Ici, chacun des deux adverbiaux temporels en tête de phrase fournit un critère sémantique pour l'interprétation non seulement de la première proposition, mais également pour les propositions subséquentes : c'est en automne que les journées raccourcissent, la nature prend de belles couleurs, le vent souffle souvent, beaucoup d'oiseaux s'envolent vers des pays plus chauds, etc. L'apparition de l'adverbial En hiver va à la fois clore cette unité discursive, appelée cadre de discours, et en ouvrir une autre en indexant les propositions qui suivent.

Dans Charolles et al. (2005), cette fonction d'indexation propre à certains adverbiaux est considérée comme une marque de cohésion discursive à côté d'autres marques de cohésion plus classiques comme les anaphores et les connecteurs. Cependant, les relations de discours établies par l'anaphore, la co-référence, les connecteurs, etc. relèvent du processus de « connexion» (cf. Halliday \& Hasan 1976, Reinhart 1981, Sanders \& Spooren 2001) alors que les cadres exploitent le processus d' «indexation ». Selon Le Draoulec et Péry-Woodley (2005 : 47), « la connexion correspond à un mode de relation « vers l'arrière », au sens où un lien se crée avec ce qui précède. L'indexation, en revanche, désigne un processus qui s'établit « vers l'avant »: l'expression indexante constitue un critère d'interprétation pour la ou les propositions qui la suivent». Les auteurs illustrent cette distinction entre «connexion » et « indexation » par le schéma suivant, repris de Charolles (1997). 
Stosic, D. (2012), « Le pouvoir cadratif des compléments introduits par à travers: des cadres de discours pas comme les autres? ». Travaux de linguistique $\mathrm{n}^{\circ} 64$, p. 55-78.

Figure 1 : connexion, notée $\mathrm{r}(c f$. Charolles 1997)

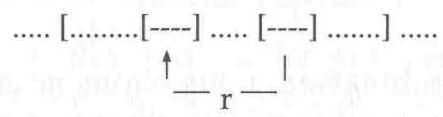

Figure 2 : indexation, notée $\mathbf{r}^{\prime}$ (ibid.)

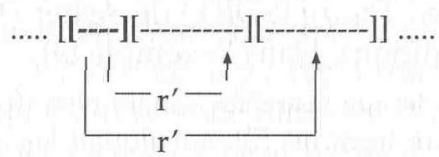

Opposition entre Relations de discours (« connexion ») et Encadrement de discours (« indexation ») (in Le Draoulec et Péry-Woodley 2005)

Les opérations discursives peuvent agir donc soit de manière rétrospective (c'est le cas de la plupart d'entre elles - anaphore, co-référence, connecteurs) soit de manière prospective (l'encadrement du discours). Les cadres reposent sur la projection d'un critère d'interprétation vers l'avant d'où leur potentiel cadratif qui leur permet de contrôler, en quelque sorte, une portion plus ou moins longue du texte. Le Draoulec et Péry-Woodley (2005:47) ajoutent que les deux modes d'organisation discursive (connexion et indexation) ne sont pas en conflit et qu'ils donnent plutôt lieu à une complémentarité.

Sans pouvoir entrer dans les détails de la question de l'importance de la position initiale pour l'encadrement du discours, précisons que le potentiel cadratif de certains adverbiaux est très étroitement lié à leur apparition en tête de phrase. Cette problématique a fait l'objet de plusieurs études (voir notamment, Charolles \& Vigier 2005 et Charolles, Colonna, Pynte \& Sarda 2004).

Un dernier point théorique important pour la suite de notre travail concerne l'opposition entre les cadres de discours «forts» et «faibles». En effet, si les premiers travaux sur l'encadrement du discours postulaient l'obligation pour un adverbial cadratif de fixer un critère d'interprétation pour chacune des propositions indexées, les études plus récentes ont mis au jour la nécessité d'assouplir l'hypothèse initiale (cf. Charolles \& Vigier 2005, Le Draoulec \& Pery-Woodley 2005, Sarda 2005, Sarda \& Stosic 2007). Plus précisément, certains adverbiaux ont effectivement un double rôle d'indexation sémantique et de découpage textuel (cf. [39] ci-dessous) alors que d'autres arrivent à délimiter des segments de texte sans indexer sémantiquement chacune des propositions qui en font partie (cf. [36]-[38] ci-dessous). Les premiers, qui cumulent une portée dite sémantique et une portée cadrative/textuelle, sont des cadres «forts», les seconds, qui n'ont qu'une portée cadrative/textuelle, sont des cadres « faibles ». 
Stosic, D. (2012), « Le pouvoir cadratif des compléments introduits par à travers: des cadres de discours pas comme les autres ? ». Travaux de linguistique $\mathrm{n}^{\circ} 64$, p. 55-78.

\subsection{Les cadres de discours introduits par les SP en à travers}

Sur les 600 occurrences des SP en à travers en tête de phrase figurant dans notre corpus, il y en a 54 qui ouvrent un cadre de discours, ce qui représente $9 \%$. Il est intéressant de noter qu'environ $60 \%$ des cadres de discours introduits par à travers relèvent du domaine de la perception [31], 16\% du domaine de la localisation [32] et $16 \%$ ont une valeur instrumentale [33]:

[31] [À travers la grille, j'apercevais la maison et, soudain, elle me parut telle que nous l'avions découverte, par ce crépuscule de janvier où notre rêve avait soudain trouvé sa voie et son visage. Les persiennes étaient presque toutes ouvertes et l'on voyait encore des rideaux à la plupart des fenêtres. Pourtant la maison semblait inanimée. Le parc était, de nouveau, clair et froid, sans profondeur. Toutes les feuilles gisaient à terre. Seul témoignage de la saison vivante, des grappes de baies rouges achevaient de se flétrir dans la ramure des sorbiers. Une feuille de platane, suspendue par un invisible fil d'araignée, tournait sur elle-même, à l'abri du petit auvent sous lequel était fixée la clochette du portail.] J'allais sonner de nouveau quand je vis Justin sortir de la maison. Il portait une blouse de peintre, toute souillée d'encre et de graisse. (Duhamel, Chronique des Pasquier)

[32] [À travers champs et par les chemins creux, nous marchions sur les hauteurs qui dominent la ville, derrière la croix et les prêtres de la paroisse. Nous chantions à pleine voix les répons aux prières faites pour attirer sur les moissons les bénédictions du ciel : «Ut nobis parcas - Te rogamus audi nos... ». (Je crois respirer encore l'air marin de ce printemps breton, le plus beau du monde.) La procession prenait l'allure d'une promenade champêtre. Sur la mousse des talus il y avait des fleurs de lait, des jonquilles, des marguerites, le chèvrefeuille sauvage embaumait. Les chemins pierreux étaient bordés de buissons de genêts. Je marchais aux côtés de mon père, heureux et fier qu'il m'eût jugé assez grand pour l'accompagner, si tôt le matin.] Nous chantions au temps de l'Avent et de Noël, agenouillés chaque soir devant la crèche que mon père dressait dans notre chambre. Nous chantions pendant le mois de mai, consacré à la Sainte Vierge. (Mohrt, La Maison du père)

[33] Le sous-sol était aussi une salle d'exposition. Mais une salle nettement plus spécialisée. Là, l'hyperréalisme avait choisi une autre cible. Plus circonscrite, plus intime. [ vingtaine de tableaux d'un graphisme quasi photographique, de beaux éphèbes aux muscles longs, aux sexes charnus, aux glands congestionnés comme des pommes d'api, pratiquaient l'amour dans des positions qui relevaient plus du fantasme que de la réalité. Corps aériens, cambrures excessives, pénétrations impossibles. Avec leurs visages de danseurs, leurs interminables yeux de biche et leurs pieds ailés, ils semblaient obéir à quelque mystérieux maître de ballet surgi des espaces infinis.] Lambert regardait. Bof. Histoire de se rebecter. (Page, Tchao pantin)

Dans la suite de notre travail, nous nous focaliserons sur les adverbiaux spatiaux, à savoir sur les cas de localisation statique et dynamique et sur les cas de perception.

\subsubsection{Typologie des cadres de discours introduits par les adverbiaux en à travers}

Compte tenu de ce qui vient d'être dit dans les paragraphes précédents, il est possible de délimiter quatre cas de figure concernant le fonctionnement discursif des SP introduits par $\grave{a}$ travers: i) emplois non-cadratifs, ii) emplois avec des cadres de discours faibles très 
Stosic, D. (2012), « Le pouvoir cadratif des compléments introduits par à travers: des cadres de discours pas comme les autres ? ». Travaux de linguistique $\mathrm{n}^{\circ} 64$, p. 55-78.

particuliers que nous appelons «cadres-fenêtres » faibles, iii) emplois avec des cadres de discours forts, particuliers eux-aussi, qualifiés de « cadres-fenêtres » forts, iv) emplois avec de véritables cadres de discours forts, proches des emplois cadratifs des adverbiaux spatiaux statiques.

\section{i) Les compléments de phrase}

Comme nous l'avons déjà dit ci-dessus, dans la grande majorité des cas le complément en à travers détaché en tête n'a pas de pouvoir cadratif, sa portée étant limitée à sa phrase d'accueil :

[34] À travers de grands bois de pins-parasols, on arrive devant une sorte de burg à massives tours d'angle qui plongent leurs fondations dans un fossé plein de gravats. L'enceinte est tout entière remplie par des édifices sans style qui tiennent à la fois d'une métairie et d'une prison. Un pont aussi barbare relie à la route la poterne d'entrée, voûte basse sans ornements. (T'Serstevens, L'Itinéraire espagnol)

[35] À travers les verres dépolis des fenêtres des chambres j'essaye de voir une ombre qui pourrait être celle de ma mère. Je monte à mon tour l'escalier en soufflant. (Guibert, Le Mausolée des amants : Journal 1976-1991)

Le SP en à travers fonctionne alors comme complément de phrase et ne participe pas à la structuration du texte.

\section{ii) Les « cadres-fenêtres » faibles}

Le complément en à travers peut introduire un cadre de discours faible d'un type très particulier : il a un pouvoir structurant au niveau du discours parce qu'il délimite un segment de texte en donnant accès à une scène de perception mais n'indexe pas sémantiquement les phrases ainsi délimitées :

[36] [À travers la porte, j'entends quelques bruits. Je reconnais la voix du président qui donne lecture de l'arrêt, mais je ne peux comprendre ses paroles. Je perçois maintenant une sorte de murmure sourd de désapprobation et de déception dans la salle. Cela signifie évidemment que je suis condamné. La lecture continue longuement. Ni les détails du jugement, ni la peine exacte ne m'intéressent. Ils ont répondu affirmativement à la question de la culpabilité, cela seul compte.] La porte s'ouvre. Quelques amis se précipitent vers moi, puis mes avocats, puis encore de nombreux confrères de Clermont-Ferrand que je ne connais pas. Tous sont navrés, consternés. (Mendès-France, S'engager)

Dans cet exemple, la porte est une entité médiane à travers laquelle la scène sera perçue. Le SP dynamique à travers la porte sert ainsi à la fois à ancrer le « point de vue », à marquer le point de départ de l'énoncé et, au-delà, à introduire toute une séquence discursive comprenant la description de la scène perçue. Comme nous l'avons déjà montré dans Sarda \& Stosic (2007), dans ce type de contextes, le complément en à travers a un rôle organisationnel mais ne fixe pas de critère sémantique commun à l'interprétation de l'ensemble des propositions réunies dans le cadre qu'il délimite : 
Stosic, D. (2012), « Le pouvoir cadratif des compléments introduits par à travers: des cadres de discours pas comme les autres ? ». Travaux de linguistique $\mathrm{n}^{\circ} 64$, p. 55-78.

«Son apparition en position initiale semble indiquer et favoriser un rôle d'organisation discursive : on accède à une scène, et grâce à cet accès on peut faire la description de la scène. L'accès et la description font un tout, un bloc textuel » (Sarda \& Stosic 2007 : 53).

Nous qualifierons de «cadres-fenêtres » ce type particulier de cadres de discours introduits par à travers, qui peuvent être faibles (cf. 36-38) ou forts (cf. 39 ci-dessous). Notons que le site dénoté par le SN complément de à travers peut correspondre soit à une entité qui donne accès à la scène qui est développée (porte, fenêtre, etc. - [38]) soit à une entité qui s'interpose entre l'observateur et la scène décrite (fumée, bruit, cloison, etc. - [37]). Que ce soit un élément qui facilite ou rende plus difficile l'accès à la scène, le site de la relation exprimée par à travers sert de médium entre l'observateur et la scène décrite dans le cadre :

[37] Si l'industrie tentait Armand, avec des recommandations, peut-être que par Maurice Perrot... après le dîner, on était allé dans un café. [À travers la fumée des cigarettes, Armand, au delà des mots maternels, s'intéressait aux voisins. Sept personnes, quatre sur la banquette, trois en face, et un gosse pâle qui ressemblait à Napoléon à Arcole. Petits boutiquiers, un melon, un mou brun, une casquette, et des femmes en noir avec des faces bouffies, blêmes. Napoléon gazouillait; il y avait un diamant à une oreille de saindoux, et soudain, dans les bras d'une des femmes, apparut un bébé dans de la laine blanche, touchant comme l'inconscience, dont les yeux profonds s'émerveillaient des lumières. Il régnait dans les moustaches avec la mousse de la bière une conversation de gros sous. Les femmes n'étaient pas les dernières à s'en mêler. Armand ne voyait plus que le tout-petit qui suçait son pouce avec conviction. (...)] (Aragon, Les Beaux quartiers)

$\mathrm{Du}$ fait de sa nature dynamique, un adverbial cadratif en à travers indexe plus difficilement les propositions subséquentes: il ne suffit donc pas de poser une relation spatiale comme dans le cas des adverbiaux statiques, il faut en plus que la relation en question puisse avoir une certaine durée, qu'elle puisse se maintenir à travers et grâce au contenu des propositions subséquentes. La position initiale n'étant pas suffisante pour le maintien de cadres-fenêtres introduits par à travers, ces derniers mobilisent souvent d'autres facteurs de cohésion discursive, comme ceux relevant des relations de connexion : les anaphores, la coréférence, les chaînes de référence, etc. (en [37], les voisins, sept personnes, quatre, trois, un gosse, petits boutiquiers, des femmes...). L'organisation de la structure informationnelle (hyper-thème, thème, rhème) du segment découpé joue également un rôle très important dans l'indexation des propositions (cf. [37] ci-dessus : les voisins, puis, sept personnes, un gosse, etc.). Sans que le recours à tous ces éléments soit de règle (cf. [38]), il est évident qu'ils facilitent le regroupement en bloc d'une suite de propositions, ce qui confère à l'adverbial en à travers détaché en tête un plus grand pouvoir structurant au niveau du discours.

[38] [À travers la vitre je voyais Murraille, debout, le combiné du téléphone entre sa joue et son épaule. Sylviane Quimphe mettait un disque sur le phono. Marcheret se versait à boire d'un geste d'automate.] 
Stosic, D. (2012), « Le pouvoir cadratif des compléments introduits par à travers: des cadres de discours pas comme les autres? ». Travaux de linguistique ${ }^{\circ} 64$, p. 55-78.

- Ils sont curieux, vos amis, ai-je remarqué. (Modiano, Les Boulevards de ceinture)

\section{iii) Les « cadres-fenêtres » forts}

Le complément en à travers peut également ouvrir un cadre de discours qui, tout en ancrant le point de vue, indexe sémantiquement plusieurs phrases. Un cadre-fenêtre fort n'est cependant possible dans le cas des adverbiaux spatiaux en à travers que si les prédicats verbaux des propositions indexées relèvent du même champ lexical :

[39] [À travers la vitre de la portière, Bernard regardait la plaine immense que traversaient lentement un homme et un cheval. Il aperçut un grand oiseau noir au dessus de l'homme et du cheval et se mit à observait attentivement les trois créatures en mouvement. Il admirait leur courage.]

Tout en présentant les traits de fonctionnement des cadres-fenêtres tels que décrits ci-dessus, les cadres en question cumulent incontestablement une portée textuelle/structurante et une portée sémantique.

\section{iv) Les cadres de discours forts}

Dans le quatrième et dernier type de contextes identifiés, le complément en à travers indexe plusieurs phrases à la manière d'un adverbial spatial statique. Il s'agit là aussi de cadres de discours forts, mais leur rôle n'est pas de donner accès à une scène. Ils fixent un cadre spatial englobant un ensemble de faits et permettent d'en faire, sur le plan textuel, une unité discursive :

[40] [À travers ces plateaux, situés en contre-bas du Massif Central, les rivières se sont frayé passage. Le Lot et la Dordogne ont buriné de profonds méandres entre des falaises rouges et grises, qui tantôt s'évasent en cirques, tantôt s'avancent en éperons sur le palier horizontal de la vallée. Des ravins latéraux à sec, des rampes en hémicycle facilitent l'accès de routes.] Les vallées sont superbes. Elles se sont creusées jusqu'au niveau des sources, par lesquelles le plateau restitue une partie des eaux qu'il a confisquées. Ces fontaines, si abondantes et si pures, avaient pour nos ancêtres gaulois un caractère sacré. (Vidal De La Blache, Tableau de la géographie de la France : 2)

Pour conclure sur cette partie, nous pouvons dire que les SP introduits par à travers ont un triple fonctionnement discursif. Ils ont la capacité à découper des segments de texte à la manière des adverbiaux cadratifs statiques, mais leur sémantisme fondamentalement dynamique fait de ces segments des cadres très particuliers. Dans la section qui suit, nous continuons leur description en nous intéressant à la façon dont ils sont clos.

\subsubsection{Clôture des cadres-fenêtres}

Une question qui mérite d'être posée à propos des cadres-fenêtres est celle de leur clôture : comment referme-t-on un cadre-fenêtre étant donné les particularités de leur 
Stosic, D. (2012), « Le pouvoir cadratif des compléments introduits par à travers: des cadres de discours pas comme les autres? ». Travaux de linguistique $\mathrm{n}^{\circ} 64$, p. 55-78.

fonctionnement sémantico-discursif? L'analyse du corpus a mis au jour plusieurs modes de clôture.

Tout d'abord, un cadre-fenêtre peut être clos par l'apparition d'un autre adverbial spatial à potentiel cadratif, comme dans l'exemple [41], où Du haut de sa cabine bloque

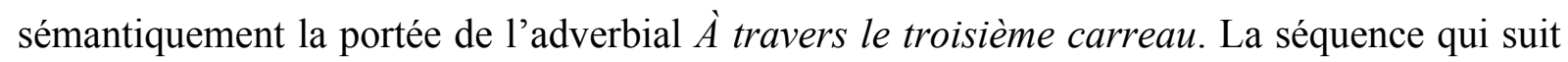
Du haut de sa cabine n'est pas un sous-cadre parce qu'elle ne peut eu aucun cas être intégrée au cadre ouvert par l'adverbial en à travers. Elle fixe un nouveau point de vue, différent du premier :

[41] Une bonne demi-heure passa ainsi. Puis Besson se leva et marcha vers la fenêtre de droite. C'était une fenêtre assez grande, plus haute que large, avec huit carreaux de verre séparés par des montants en bois marron. Le quatrième carreau à partir du bas était fêlé dans toute sa longueur, et le sixième tremblait légèrement. [À travers le troisième carreau, Besson regarda le spectacle des maisons et de la chaussée. Sous la pluie fine, les silhouettes rapetissées marchaient dans tous les sens, vieillards, enfants, hommes, femmes. Les voitures glissaient péniblement vers le carrefour, s'arrêtaient, puis repartaient en changeant de vitesse. De temps à autre il y avait des coups de klaxon, des sortes de hûûûûut plutôt brefs qui ne soulevaient pas d'écho. C'était un spectacle familier, presque paisible. (Et pourtant, c'était quand même inquiétant. On était en sécurité provisoire, en haut de cet immeuble. Le flot de l'agitation vous cernait peu à peu, comme ça, sans en avoir l'air. Les tourbillons bruyants usaient sans trêve les murs de la maison, ils arrachaient des bribes de plâtre, des petits cailloux, des plaques de peinture ocre. Les hommes qui rôdaient sur les trottoirs n'étaient pas inoffensifs. Dans leurs têtes baissées, ils cachaient des pensées meurtrières; il suffisait de vraiment peu de chose, une révolution par exemple, un simple mouvement de foule en colère. Ils passeraient comme des fourmis voraces, se masseraient sous les fenêtres, criant, tendant les poings. Ils hurleraient: «à mort! à mort!» Ils viendraient tous par les escaliers, ils défonceraient les portes, et ils frapperaient, ils frapperaient sans pitié à grands coups de lames de rasoir, jusqu'à ce que les têtes se séparent et tombent, béantes d'une large plaie rouge d' où s'échappe la vie. Il ne fallait pas y penser. Il ne fallait plus voir des choses que leur apparence, et jouer avec elles tout le temps, comme avec des osselets.) Avec une espèce d'obstination, Besson se mit à compter les voitures qui passaient. Il prit un bout de papier et un crayon à bille; après quelques minutes, il arriva au résultat suivant: Citroën... 14 Renault... 51 Dauphine... 294 CV... 12 R4, R8... 10 Peugeot (403, 404).. 25 Panhard... 5 Simca... 6 Fiat... 9 Alfa-Romeo... 1 Volkswagen... 1 Ford... 1 Porsche... 1 Dodge Dart... 1 Volvo... 1 Marques inconnues... 3 Le long des trottoirs, les

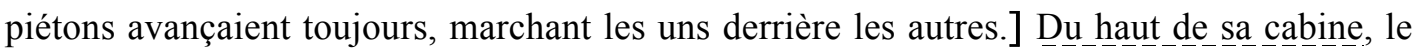
front collé à la vitre, François Besson les regarda attentivement. Il vit des femmes jeunes, vêtues d'imperméables rouge et noir, les unes la tête nue, d'autres portant des chapeaux ou des foulards. (Le Clézio, Le Déluge)

Ensuite, le maintien du cadre-fenêtre peut être bloqué par des facteurs purement sémantiques, sans marquage formel explicite, comme dans l'exemple suivant, où la description passe du domaine de la perception visuelle à celui de la perception olfactive :

[42] - Tu n'en as plus du tout (de doublezons)? demanda Chick.

- À peine... répondit Colin. J'ai payé d'avance pour la montagne et pour les fleurs parce que je ne veux rien ménager pour tirer Chloé de là. Mais, à part ça, les choses vont mal d'ellesmêmes.

Chick avait fini sa saucisse. 
Stosic, D. (2012), « Le pouvoir cadratif des compléments introduits par à travers: des cadres de discours pas comme les autres? ». Travaux de linguistique $\mathrm{n}^{\circ} 64$, p. 55-78.

- Viens voir le couloir de la cuisine, dit Colin!

- Je te suis, dit Chick.

[À travers les vitres, de chaque côté, on distinguait un soleil terne, blafard, semé de grandes taches noires, un peu plus lumineux en son centre. Quelques maigres faisceaux de rayons réussissaient à pénétrer dans le couloir, mais, au contact des carreaux de céramique autrefois si brillants, ils se fluidifiaient et ruisselaient en longues traces humides.] Une odeur de cave émanait des murs. (Vian, L'Écume des jours)

Enfin, le changement de relation entre l'observateur et le site peut également mettre fin à un cadre-fenêtre. En effet, le fait de quitter l'endroit ou d'écarter l'objet donnant accès à la scène (en ouvrant la porte, par exemple) clôt en général le cadre, le site médian perdant ainsi sa fonction de médium. Dans l'exemple [43], c'est ça qui empêche le complément en à travers d'étendre sa portée au-delà de la phrase, et de façon analogue, en [44], le fait que l'observateur quitte son point d'observation en montant dans sa voiture, clôt le cadre :

[43] À travers la vitre de la cabine je vis Anglarès qui s'inclinait en souriant le récepteur à la main. J'ouvris la porte. "Qu'est-ce qu'il y a ? » me demanda-t-il. (Queneau, Odile)

[44] À côté de la jeune femme, il y avait le cadavre de Lorque, un peu plus loin celui de Sonia. Aimée se leva, gagna en titubant la Mercedes et éteignit les phares. [À travers la nuit qui grisaillait, elle distinguait d'un côté le bassin et les chalutiers à quai, et Bléville au-delà où dormaient les honnêtes gens ; et de l'autre côté elle devinait l'autre bassin et puis la pente sur quoi s'étendent les faubourgs ouvriers avec leurs rues qui s'appellent Jean Jaurès, Gagarine, Libération.] Aimée monta dans la Mercedes. Les clés étaient au contact. Elle démarra. (Manchette, Fatale)

Les trois modes de clôture des cadres-fenêtres mentionnés sont ceux qui apparaissent le plus souvent dans notre corpus, mais les possibilités de clôture sont certainement loin d'être épuisées.

\section{Conclusion}

L'objectif de cet article était d'examiner la capacité des SP introduits par la préposition dynamique à travers à apparaître en position initiale de la phrase et puis à fonctionner sur le plan textuel en découpant des cadres de discours. Ainsi, nous avons pu montrer que les adverbiaux spatiaux en à travers, tout en étant dynamiques, peuvent remonter en tête de phrase et, à partir de là, avoir un pouvoir structurant sur le plan textuel en introduisant un type particulier de cadres de discours. Grâce à leur sémantisme, les adverbiaux cadratifs en à travers ont pour particularité de donner accès à une scène, scène qui fera l'objet par la suite d'une description plus ou moins développée. L'adverbial et la description qui est sous sa portée font un bloc textuel, qualifié de «cadre-fenêtre ». La plupart des cadres-fenêtres sont des cadres de discours faibles, mais les cadres forts sont également attestés. Enfin, c'est dans les contextes perceptuels que les SP en à travers se prêtent le plus facilement au fonctionnement discursif. 
Stosic, D. (2012), « Le pouvoir cadratif des compléments introduits par à travers: des cadres de discours pas comme les autres ? ». Travaux de linguistique $\mathrm{n}^{\circ} 64$, p. 55-78.

Quant à la capacité des SP en à travers à ouvrir des cadres de discours, elle s'avère en fin de compte relativement limitée: d'après nos données, même pas un dixième des occurrences des SP en à travers en position initiale aurait un fonctionnement discursif. Ce chiffre paraît d'autant plus bas quand on sait que ces adverbiaux, à cause de leur sémantisme dynamique et de leur statut argumental ambigu (excepté dans la construction transitive directe), remontent difficilement en tête de phrase : une occurrence sur vingt, dans notre corpus. Notons cependant que, même s'il est évident que les adverbiaux spatiaux statiques détachés en tête ouvrent plus facilement et plus souvent les cadres de discours, on ne dispose pas vraiment de données statistiques qui permettent de comparer leur capacité à introduire des cadres à celle des adverbiaux dynamiques en à travers. Il serait intéressant de contraster les deux à partir du même type de données et selon la même méthodologie.

Cette étude met également en évidence que les SP en à travers à valeur instrumentale détachés en tête de phrase peuvent avoir une portée cadrative. Une des pistes de recherche serait d'examiner en détails des points communs entre les cadres spatiaux et instrumentaux, étant donné que les premiers sont très particuliers à cause du sémantisme de à travers. Une éventuelle ressemblance ou analogie confirmerait, par un effet de retour, l'unité sémantique de la préposition à travers. 
Stosic, D. (2012), « Le pouvoir cadratif des compléments introduits par à travers: des cadres de discours pas comme les autres? ». Travaux de linguistique $\mathrm{n}^{\circ} 64$, p. 55-78.

\section{Bibliographie}

Aurnague M., 2000, «Entrer par la petite porte, passer par des chemins de traverse: à propos de la préposition par et de la notion de "trajet" », Carnets de grammaire, 7.

Aurnague M. et Stosic D., 2002, « La préposition par et l'expression du déplacement : vers une caractérisation sémantique et cognitive de la notion de "trajet" », Cahiers de Lexicologie, 81/2, p. 113-139.

BORILLO A., 1998, L'expression de l'espace en français, Paris, Ophrys.

BORILlO A., 2007, «On the spatial meaning of contre in French : The role of entities and force dynamics ", in Aurnague M., Hickmann M. et Vieu L. (eds), The categorization of spatial entities in language and cognition, Amsterdam Philadelphia, John Benjamins, p. 53-70.

BonAmi O., 1999, Les constructions du verbe : le cas des groupes prépositionnels argumentaux. Analyse syntaxique, sémantique et lexicale. Thèse de doctorat, Université Paris VII.

Charolles M., 1997, «L'encadrement du discours : univers, champs, domaines et espaces », Cahiers de Recherche Linguistique, 6, p. 1-73.

Charolles M., Le Draoulec A., Pery-Woodley M.-P. et Sarda L., 2005, « Temporal and spatial dimensions of discourse organisation », Journal of French Language Studie,s 15/2, p. 115-130.

Charolles M. et Pery-Woodley M.-P. (éds), 2005, Les adverbiaux cadratifs, Langue française 148.

Charolles M. et Vigier D., 2005, « Les adverbiaux en position préverbale : Portée cadrative et organisation des discours », Langue Française, 148, p. 9-30.

Charolles M., Colonna S., Sarda L., \& Pynte J., 2004, The effect of the initial position of spatial adverbials on the understanding of texts. Communication orale, Workshop Relations from sentence to discourse: from form to processing, Aix en Provence, September 20-21.

Dendale P., 2001, «L'emploi spatial de contre : propositions pour un traitement unifié », Travaux de linguistique, 42-43, p. 229-239.

DOMINICY M. \& MARTIN F. (2005), « À travers et au travers de. Des emplois perceptuels aux emplois spatiaux », Recherches Linguistiques, 27, 151-190. 
Stosic, D. (2012), « Le pouvoir cadratif des compléments introduits par à travers: des cadres de discours pas comme les autres? ». Travaux de linguistique nº4, p. 55-78.

FlauX N. et Stosic D., 2011, « Noms d'idéalités, prépositions et temporalité », in ARJOCAIeremia E., Avezard-Roger C., Goes J., Moline E. et Tihu A. (éds), Temps, aspect et classes de mots : études théoriques et didactiques, Arras, Artois Presses Université, p. $155-177$.

Flageul V. 1997, Description sémantico-cognitive des prépositions spatiales du français. Thèse de doctorat, Université Paris IV.

Halliday M.A.K. et Hasan R., 1976, Cohesion in English, London, Longman.

HusSERL E., [1929] 1970a, Logique formelle et Logique transcendantale, Paris, P.U.F.

Husserl E., [1938] 1970b, Expérience et Jugement. Recherches en vue d'une généalogie de la logique, Paris, PUF.

KWON-PAK S. N. 1997, Les prépositions spatiales: sur quelques emplois de la préposition "par", Thèse de doctorat, Université Strasbourg 2.

LAGAE V., à paraître, «Marqueurs du point de départ spatial et temporel antéposés : une comparaison de depuis, dès et à partir de », Corela (numéro thématique).

LAUR D., 1993, «La relation entre le verbe et la préposition dans la sémantique du déplacement », Langages, 110, p. 47-67.

Le Draoulec A. \& Pery-Woodley M.-P., 2005, «Encadrement temporel et relations de discours », Langue Française, 148, p. 45-60.

Papahagi C., 2005, Les prépositions de la trajectoire en français et en roumain : étude synchronique et diachronique. Thèse de doctorat, Université Paris 3.

Plungian V., 2002, « $\grave{A}$ travers et les autres mots de sa famille : grammaticalisation et polysémie », SCOLIA, 15, p. 123-132.

Prevost S., 2003, «Les compléments spatiaux : du topique au focus en passant par les cadres », Travaux de Linguistique, 47, p. 51-78.

REINHART T., 1981, «Pragmatics and linguistics: an analysis of sentence topics », Philosophica, 27, p. 53-94.

SANDERS T. et SPOOREN W., 2001, «Text representation as an interface between language and its users », in SAnders T., Schilperoord J. et Spooren W. (eds), Text Representation - Linguistic and psycholinguistic aspects, Amsterdam, John Benjamins.

SARDA L., 2005, «Fonctionnement des cadres spatiaux dans les résumés de films », Langue Française, 148, p. 61-79. 
Stosic, D. (2012), « Le pouvoir cadratif des compléments introduits par à travers: des cadres de discours pas comme les autres ? ». Travaux de linguistique $\mathrm{n}^{\circ} 64$, p. 55-78.

SARDA L. et Stosic D., 2007, «Les compléments spatiaux dynamiques détachés en tête : analyse des compléments en par et à travers dans la perspective de l'encadrement du discours », in FlaUX N. et STOSIC D. (éds), Les constructions détachées : entre langue et discours, Arras, Artois Presses Université, p. 41-56.

SpANG-HANSSEN E., 1963, Les prépositions incolores du français moderne, Copenhague, Gads Forlag.

Stosic D., 2002, "Par” et “à travers" dans l'expression des relations spatiales: comparaison entre le français et le serbo-croate. Thèse de doctorat, Université de Toulouse-Le Mirail.

Stosic D., 2007a, «The Prepositions par and à travers and the Categorization of Spatial Entities in French », in Aurnague M., Hickmann M. et Vieu L. (eds), The categorization of spatial entities in language and cognition, Amsterdam Philadelphia, John Benjamins, p. 71-91.

STOSIC D., 2007b, « Le sens spatial de la préposition à travers en français », communication au Colloque international Autour de la préposition, Université de Caen, Caen (20-22 septembre 2007).

Stosic D., 2009, « Comparaison du sens spatial des prépositions à travers en français et kroz en serbe », Langages, 173, p. 15-33.

Stosic D. et Flaux N., 2012, « Les noms d'idéalités sont-ils polysémiques ? ». In SAUSSURE L. DE et RiHS A. (éds), Etudes de sémantique et pragmatique françaises, Bern, Peter Lang, p. 167-190.

Trésor de la langue française informatisé (TLFi), http://atilf.atilf.fr/tlf.htm.

VANDELOISE C., 1986, L'espace en français : sémantique des prépositions spatiales, Paris, Le Seuil.

VANDELOISE C., 2003, «Les usages spatiaux de la préposition contre ». Recherches Linguistiques, 26, p. 15-44.

VAndeloise C., 2005, «Contre et la rencontre atypique ». Recherches Linguistiques, 27, p. 121-149.

WeInRICH H., 1989, Grammaire textuelle du français, Paris : Didier/Hatier. 